\title{
Optimizing cell size in Pico-cell networks
}

\author{
Sreenath Ramanath ${ }^{1}$, Eitan Altman ${ }^{1}$, Vinod Kumar ${ }^{2}$ and Merouane Debbah ${ }^{3}$ \\ ${ }^{1}$ INRIA, Sophia Antipolis, FRANCE. ${ }^{2}$ Alcatel-Lucent Bell Labs, Paris, FRANCE. ${ }^{3}$ Supelec, Paris, FRANCE
}

\begin{abstract}
In this paper, we present a systematic study of the uplink capacity and coverage of pico-cell wireless networks. Both the one dimensional as well as the two dimensional cases are investigated. Our goal is to compute the size of pico-cells that maximizes the spatial throughput density. To achieve this goal, we consider fluid models that allow us to obtain explicit expressions for the interference and the total received power at a base station. We study the impact of various parameters on the performance: the path loss factor, the spatial reuse factor and the receiver structure (matched filter or multiuser detector). We relate the performance of the fluid models to that of the original discrete system and show that the fluid model provides a bound for the discrete one.
\end{abstract}

Index Terms-Cellular networks, Pico-cell networks.

\section{INTRODUCTION}

Pico-cell based wireless networks are gaining wide popularity to provide the end user with uniform coverage, symmetry and throughput [12], [13]. Existing cellular networks like GSM and WiMAX are designed to cope with to large coverage areas, which does not achieve the expected throughput to ensure seamless mobile broadband in the up-link (UL), as one moves farther away from the BS. This is due to an increase in the Inter-cell interference (ICI) as well as constraints on the transmit power of the mobiles. Another limitation of the macro-cell approach is the poor indoor penetration.

Advanced signal processing techniques, expanded use of spectrum, improved modulation and coding are some of the techniques used to scale the capacity of a system. But, for a given radio architecture, efficient frequency reuse by dividing a large (macro) cell into number of small (pico) cells is one of the most effective ways to increase system capacity [13]. While improving the overall throughput achievable in the macro-cell, this also brings down deployment costs. This is a step closer to any-place, any-time, any-device mobile broadband access.

In a pico-cell, the shorter transmission distance coupled with lower transmit power, enhances both capacity as well as the Signal to Interference Noise Ratio (SINR) achievable within the cell. But, a designer or a system architect would like to answer questions such as: What is the optimum number of cells that one would want to divide the macro-cell?, What is the optimum cell size which maximizes the throughput achievable at a pico-cell?. Does the receiver configuration matter? How is throughput affected when one moves from a deployment of pico-cells on a street (1D) to a deployment in a office space or shopping mall (2D)?. What if the entire cell is located indoors? What happens if the pico-cell BS is within the building or located outside? What is the implication of frequency reuse on the throughput achievable?

In this contribution, we try to address several of these questions. In particular, we derive explicit expressions for the up-link (UL) SINR and throughput for simple 1D and 2D models and analyze the achievable throughput as a function of the cell size, coverage, etc.

The goal of this work is to develop an analytical framework that can be used for preliminary dimensioning purposes in planning the pico-cell network and thus provide an insight for answering the above questions. We derive closed form expressions of useful performance metrics considering free space path loss. We plan to incorporate refined models that include detailed propagation models, fading and mobility in subsequent work. Since a detailed model may require long simulations and/or heavy numerical computations, we believe that the insight brought from our approach can be used as inputs for a more detailed model: it can give an idea as to what range of parameters one can restrict the search for optimal performance.

We begin our study with the computation of the received power in single frequency and frequency reuse in Section II. Next, in Section III, we derive expressions for the throughput for both the modes with base-station (BS) receivers using the matched filter and multi-user detector. Using expressions derived in Section III, we study the impact of cell size on the achievable throughput at the BS in Section IV and propose a simple optimization criteria for both receiver configurations. In Section V, we analyze the impact of cell size on the throughput in indoor scenarios. We derive expressions for the received power and throughput for a 2D model in Section VI and use it to study the throughput as a function of cell size. Finally in Section VII, we look at the trade-off between capacity and coverage for the models presented in previous sections. We conclude our observations in Section VIII.

\section{A. Related work}

We use a fluid model approach similar to the one used in [1], [5]. The simplicity of the fluid model approach eases laborious and time consuming simulations. Typically the cell structures are uniformly placed base-stations on a line segment (1D) or hexagons (2D). This model is simplistic. One can also use a Poisson-Voronoi model. Both models are discussed for example in [14]. The principal behind the hexagon approach is to construct a disc with an equivalent area similar to the hexagon (OR hexagons of interest). While in the PoissonVoronoi model, the base station locations and cell sizes are random. These two are extreme and complementary architec- 
tures: The hexagonal model represents perfectly structured networks, whereas the Poisson-Voronoi model takes into account irregularities of real networks in a statistical way. We shall treat in detail the hexagonal model in this paper.

An alternative "fluid" model has been introduced in [2], [3] to study the performance and optimal cell size for CDMA networks. The model includes fading, and the fluid limits are those obtained when the density of mobiles become large. The main tool there has been random matrix theory. The approach in [2], [3] requires complete homogeneity within each cell: the gain from a mobile to a base station does not depend on the location of the mobile. In contrast, in our paper we take into account the detailed impact of the distance on the channel gains. In [6] the authors address a related problem of optimizing the spectral efficiency of cellular indoor wireless networks by adjusting the location and power of the base-stations. They apply both continuous and combinatorial approaches to find a solution to the optimization problem.

\section{RECEIVED POWER COMPUTATION}

We compute the total power received at the base station for single frequency and frequency reuse modes as a function of the cell size. The single frequency or frequency reuse modes can employ single carrier or OFDM modulation for transmission.

\section{A. Single frequency $(S F)$}

Assume a single frequency deployment. There is a uniform density of mobiles on the line $\mathcal{L}=((-\infty, 0),(\infty, 0)$, each transmitting at a unit power (similar to the fluid model approach used in [1], [2]). There are base stations (BS) located at $(n L, 1), n=\ldots,-1,0,1, \ldots$. Since we consider picocells, we explicitly include in the geometric model the vertical distance (normalized to one) of the base station (which could be negligible in large cells). Let $\alpha$ represent the attenuation factor or path loss factor in the given wireless environment (for practical values of alpha one can refer for ex. to [10]). Thus the power received at the BS from a mobile at a distance of $x$ is equal to $\left(1+x^{2}\right)^{-\alpha / 2}$.

Denote $\beta_{1}=(\alpha-1) / 2$ and $\beta_{2}=(\alpha+1) / 2$. The total power received at the $\mathrm{BS}$ is

$$
P_{b s}^{t o t}=\int_{-\infty}^{\infty}\left(1+x^{2}\right)^{-\alpha / 2} \mathrm{~d} x=\frac{\sqrt{\pi} \Gamma\left(\beta_{1}\right)}{\Gamma(\alpha / 2)}
$$

In particular, we have in Table I explicit expressions for some integer valued $\alpha$ 's:

TABLE I

TOTAL RECEIVED POWER AS A FUNCTION OF $\alpha$

\begin{tabular}{|c|ccccc|}
\hline$\alpha$ & 2 & 3 & 4 & 5 & 6 \\
\hline $\begin{array}{c}\text { Total received } \\
\text { power }\end{array}$ & $\pi$ & 2 & $\frac{\pi}{2}$ & $\frac{4}{3}$ & $\frac{3}{8} \pi$ \\
\hline
\end{tabular}

1) Power received at the $B S$ in $C_{0}$ : Define the cell $C_{0}$ to be the segment $[-L / 2, L / 2]$ on the line $\mathcal{L}$. The total power received at the $\mathrm{BS}$ in $C_{0}$ from mobiles in $C_{0}$ is

$$
\begin{aligned}
P_{b s}^{C_{0}}(L)= & \int_{-L / 2}^{L / 2}\left(1+x^{2}\right)^{-\alpha / 2} \mathrm{~d} x \\
= & \frac{1}{\beta_{1}}\left(\frac{L}{2}\right)^{1-\alpha} \text { hypergeom }\left(\left[\frac{\alpha}{2}, \beta_{1}\right], \beta_{2},-\frac{4}{L^{2}}\right) \\
& -\frac{\pi^{3 / 2} \sec \left(\frac{\pi \alpha}{2}\right)}{\Gamma\left(\frac{\alpha}{2}\right) \Gamma\left(\frac{3-\alpha}{2}\right)}
\end{aligned}
$$

For the special case of $\alpha=2$ this simplifies to

$$
P_{b s}^{C_{0}}(L)=\pi+i \log \left(\frac{L+2 i}{L-2 i}\right)
$$

Where $L$ is the cell size and $i=\sqrt{-1}$. In figure (2) we depict this case for $L$ taking the values from 0.01 to 10 for $\alpha=2,4$. We can see that $P_{b s}^{C_{0}}(L) \rightarrow P_{b s}^{t o t}$ as $\mathrm{L}$ increases.

\section{B. Frequency reuse $(F R)$}

We consider some time slot and a given frequency and assume that this same frequency is not used at the same time slot in all cells: it is separated by $m-1$ cells. In figure (1), we present a typical frequency allocation for a one dimensional (1D) case. $m=1$ is the single frequency case, whereas, any $m>1$ represents frequency reuse. We show in the figure a typical reuse- 3 case. i.e, every third cell uses the same frequency.

The total power received at the $\mathrm{BS}$ is

$$
P_{b s}^{t o t}=P_{b s}^{C_{0}}(L)+\sum_{i=-\infty, i \neq 0}^{\infty} P_{b s}^{C(i m)}(L)
$$

where $P_{b s}^{C(j)}(L)$ is the power received at the BS from cell $j$ that has size $L$. We note that

$$
\begin{aligned}
P_{b s}^{C(i m)}(L)+P_{b s}^{C(-i m)}(L)= & P_{b s}^{C_{0}}((2 m+1) L) \\
& -P_{b s}^{C_{0}}((2 m-1) L)
\end{aligned}
$$

\section{THROUGHPUT}

We compute the total achievable throughput at a cell in the case where the BS receiver uses a matched filter or employs a multi-user detection scheme like successive interference cancellation.

\section{A. Matched filter $(\mathrm{MF})$}

We model the power received at BS in cell $C_{0}$ from a mobile at $x$ as the total power received from $[x, x+d x]$, i.e. $d P(x)=$ $\left(1+x^{2}\right)^{-\alpha / 2} \mathrm{~d} x$. We use the Shannon capacity to compute the throughput while treating the interferences from all other mobiles at the same frequency and time as noise. Using a 
detection scheme based on the matched filter, the achievable throughput from the mobile at $x$ is

$$
d \theta(x)=\log \left(1+\frac{d P(x)}{\sigma^{2}+P_{b s}^{t o t}}\right)
$$

where, $\sigma^{2}$ is the noise power.

Since the quantity $\frac{d P(x)}{P_{b s}^{t o t}}<<1$, we use $\log (1+x) \approx x$ and rewrite

$$
d \theta(x)=\frac{d P(x)}{\sigma^{2}+P_{b s}^{t o t}}
$$

Hence the total throughput at the cell is given by

$$
\Theta(L)=\frac{\int_{-L / 2}^{L / 2} d P(x) d x}{\sigma^{2}+P_{b s}^{t o t}}=\frac{P_{b s}^{C_{0}}(L)}{\sigma^{2}+P_{b s}^{t o t}} .
$$

and the throughput density is given by

$$
\Psi_{M F}(L)=\frac{\Theta_{C}}{L}=\frac{P_{b s}^{C_{0}}(L) / L}{\sigma^{2}+P_{b s}^{t o t}} .
$$

Let $L^{*}$ denote the cell size which maximizes the throughput density.

Lemma 1: In the case of the matched filter, the throughput density is maximized by taking base stations as dense as possible. i.e, $L^{*} \rightarrow 0$.

Proof : From equation (8),

$$
\begin{aligned}
L^{*} & =\arg \max _{L} \frac{P_{b s}^{C_{0}}(L) / L}{\left(\sigma^{2}+P_{b s}^{t o t}\right)} \\
& =\arg \max _{L} P_{b s}^{C_{0}}(L) / L \\
& =\arg \max _{L}\left(\frac{1}{L} \int_{0}^{L}\left(1+x^{2}\right)^{\frac{-\alpha}{2}} d x\right)
\end{aligned}
$$

as $\sigma^{2}$ and $P_{b s}^{t o t}$ are independent of $L$. Clearly,

$$
\int_{0}^{L} 1^{\frac{-\alpha}{2}} d x>\int_{0}^{L}\left(1+x^{2}\right)^{\frac{-\alpha}{2}} d x>\int_{0}^{L}\left(1+L^{2}\right)^{\frac{-\alpha}{2}} d x
$$

and hence,

$$
1>\frac{P_{b s}^{C_{0}}(L)}{L}>\left(1+L^{2}\right)^{\frac{-\alpha}{2}} .
$$

From the above, it is easy to see that,

$$
\begin{aligned}
& \frac{P_{b s}^{C_{0}}(L)}{L}<1 \text { if } L>0, \\
& \frac{P_{b s}^{C_{0}}(L)}{L} \rightarrow 1 \text { as } L \rightarrow 0 .
\end{aligned}
$$

And thus, $L^{*}=0$.

\section{B. Multi-user detection (MD)}

We assume that all the signal received at $\mathrm{BS}$ in cell $C_{0}$ from mobiles out of $C_{0}$ are considered noise; however within $C_{0}$, some multi-user detection scheme that maximizes the cell throughput is used. For example, successive interference cancellation is assumed. Then the SINR is given by

$$
S I N R=\frac{P_{b s}^{C_{0}}(L)}{\sigma^{2}+P_{b s}^{t o t}-P_{b s}^{C_{0}}(L)} .
$$

and the throughput achievable using the Shannon capacity limit with the multi-user detection constraint [11]

$$
\Theta(L)=\log (1+S I N R)
$$

and the throughput density is

$$
\Psi_{M D}(L)=\frac{\Theta}{L}=\frac{\log \left(1+S I N R_{C}\right)}{L}
$$

Lemma 2: In the case of multi-user detection, the cell size which maximizes throughput density is such that $L^{*}>0$

Proof : From equation (11),

$$
L^{*}=\arg \max _{L} \frac{1}{L} \log \left(1+\frac{P_{b s}^{C_{0}}(L)}{\sigma^{2}+P_{b s}^{t o t}-P_{b s}^{C_{0}}(L)}\right)
$$

Let $\sigma^{2}+P_{b s}^{t o t}=K$. By monotonicity of log function,

$$
L^{*}=\arg \max _{L}\left(\frac{K}{K-P_{b s}^{C_{0}}(L)}\right)^{\frac{1}{L}}
$$

Clearly,

$$
L>P_{b s}^{C_{0}}(L)>L\left(1+L^{2}\right)^{\frac{-\alpha}{2}}
$$

and hence,

$$
\left(\frac{K}{K-L\left(1+L^{2}\right)^{\frac{-\alpha}{2}}}\right)^{\frac{1}{L}}>\left(\frac{K}{K-P_{B S}^{C_{0}}(L)}\right)^{\frac{1}{L}}>\left(\frac{K}{K-L}\right)^{\frac{1}{L}}
$$

From the above, it is easy to see that

$$
\left(\frac{K}{K-P_{B S}^{C_{0}}(L)}\right)^{\frac{1}{L}}>1 \text { if } L>0,
$$

$$
\left(\frac{K}{K-P_{B S}^{C_{0}}(L)}\right)^{\frac{1}{L}} \rightarrow 1 \text { as } L \rightarrow 0 .
$$

Thus there is an $L^{*}>0$, which maximizes the throughput density.

Computation of $L^{*}$ : By virtue of Lemma 2, $L^{*}>0$, i.e., the maximizer is not at the boundary point and the objective function is clearly a differentiable function. Hence, the cell size which maximizes the throughput density is a zero of the derivative $d \Psi_{M D} / d L$ and hence is a zero of

$$
\Omega\left(L^{*} ; \alpha, \sigma^{2}\right)=0
$$


where

$$
\begin{aligned}
\Omega\left(L ; \alpha, \sigma^{2}\right) & :=L\left(1+\frac{L^{2}}{4}\right)^{\frac{-\alpha}{2}} \\
& -\left(\sigma^{2}+P_{b s}^{t o t}-P_{b s}^{C 0}\right) \log \left(\frac{\sigma^{2}+P_{b s}^{t o t}}{\sigma^{2}+P_{b s}^{t o t}-P_{b s}^{C 0}}\right)
\end{aligned}
$$

Finding an explicit expression for $L^{*}$ is not easy. We outline a simple procedure to compute $L^{*}$ iteratively.

$$
L_{k+1}=L_{k}+\epsilon \Omega\left(L_{k} ; \alpha, \sigma^{2}\right)
$$

$L_{0}>0$ is the initial value for the first iteration and $L^{*}$ is the converged value. One can confirm the above iteration has converged whenever the error $\left|L_{k+1}-L_{k}\right|<\mu$ for some small enough positive constant $\mu$.

For example, with $\alpha=2, \sigma^{2}=1, L_{0}=0.1$ and $\epsilon=0.1$, we converge to $L^{*}=0.85$ in 150 iterations. This matches with the optimal $(\max )$ value for the curve labeled $S F \_M D$ in figure (5).

Asymptotic Approximations for $L^{*}$ in Pico-cells: We derive approximations for $L^{*}$ as the path-loss factor $\alpha$ converges to 0 or to $\infty$. We recall from equation (11),

$$
L^{*}=\arg \max _{L \in\left[0, L_{\text {max }}\right]} \frac{1}{L} \log \left(1+\frac{P_{b s}^{C_{0}}(L)}{\sigma^{2}+P_{b s}^{t o t}-P_{b s}^{C_{0}}(L)}\right) .
$$

In the above, $L_{\max }$ represents the maximum cell size that we can design. Since we are dealing with pico-cells $L_{\max }$ itself is a small number and hence $P_{b s}^{C_{0}}(L) \ll P_{b s}^{t o t}$. So we can use the approximation $\log (1+x) \approx x$ and re-write

$$
L^{*}=\arg \max _{L \in\left[0, L_{\max }\right]} \frac{1}{L}\left(\frac{P_{b s}^{C_{0}}(L)}{K_{\alpha}-P_{b s}^{C_{0}}(L)}\right),
$$

where $K_{\alpha}:=\sigma^{2}+P_{b s}^{t o t}$.

For large values of $\alpha$ : By bounded convergence theorem as $\alpha \rightarrow \infty, P_{b s}^{C_{0}}(L)-L\left(1+L^{2}\right)^{-\alpha / 2} \rightarrow 0$. Therefore for large values of $\alpha, L^{*} \approx \arg \max _{L} \frac{1}{L}\left(\frac{L\left(1+L^{2}\right)^{-\alpha / 2}}{K_{\alpha}-L\left(1+L^{2}\right)^{-\alpha / 2}}\right)$ or $L^{*}$ approximately solves

$$
\left.\frac{d\left(\frac{\left(1+L^{2}\right)^{-\alpha / 2}}{K_{\alpha}-L\left(1+L^{2}\right)^{-\alpha / 2}}\right)}{d L}\right|_{L=L^{*}}=0 .
$$

Solving this yields

$$
L^{*} \approx \frac{1}{\alpha K_{\alpha}} \text { for large values of } \alpha .
$$

For small values of $\alpha:$ By bounded convergence theorem again as $\alpha \rightarrow 0, P_{b s}^{C_{0}}(L) \rightarrow L$. Therefore, $L^{*} \approx$ $\arg \max _{L}\left(\frac{1}{K_{\alpha}-L}\right)$ for small values of $\alpha$. Hence

$$
L^{*} \approx L_{\max } \text { for small values of } \alpha .
$$

In figure (9), we plot $L^{*}$ as a function of $\alpha$ computed using equations (15), (16) and compare it with the value of $L^{*}$ computed via numerical simulations. We see that the numerically evaluated values lie within the two bounds. Also, for smaller values of $\alpha$, the simulation results are closer to the $\alpha$ small bound and for larger values, the simulation results are closer to the $\alpha$ large bound.

\section{Comments on the fluid approach}

So far we have been working with a fluid model which had the advantage (over a more detailed discrete stochastic model) of being sufficiently simple to allow us to obtain explicit expressions for performance measures related to the system capacity. We now address the question of the validity of the fluid approximation: can we estimate what these expressions say on the original discrete system? To address the question we first define our stochastic model. We assume a Poisson arrival process with constant intensity.

Assume that mobiles are located according to a uniform Poisson point process $X=\left\{X_{i}\right\}$ with intensity $\lambda$ which we normalize to one unit. $N_{x}(A)$ denotes the number of points of the process $X$ in a set $A$. Consider first the single frequency setting of Section II-A. Then the total power received at the BS is given by $\sum_{i=-\infty}^{\infty}\left(1+X_{i}^{2}\right)^{-\alpha / 2}$ and its expectation equals precisely to $\int_{-\infty}^{\infty}\left(1+x^{2}\right)^{-\alpha / 2} \mathrm{~d} x$, for which equation (1) provides an explicit expression (for integer $\alpha$ 's).

The power and the interferences received at the BS according to the fluid approximation are thus precisely the expectation of those corresponding to the discrete model. In particular, the remaining equations of Section II-A also hold for the expectations of the corresponding objects in the discrete model.

The total throughput of the cell is then given by equation (7) and its expectation satisfies $E[\Theta(L)]=$ $E\left[P_{b s}^{C_{0}}(L)\right] E\left[\frac{1}{\sigma^{2}+P_{b s}^{t o t}}\right] \geq \frac{E\left[P_{b s}^{C_{0}}(L)\right]}{\sigma^{2}+E\left[P_{b s}^{t o t}\right]}$ where the first equality follows from the independence properties due to the Poisson assumption on the location of mobiles, and the last step follows by Jensen's inequality. We conclude that the throughput results of the fluid model provide lower bounds to those of the discrete model.

\section{IMPACT OF CELL SIZE ON THROUGHPUT}

In this section, we use the expressions obtained in the previous sections to study the impact of the cell size on throughput. This analysis will allow us to optimize the cell size for the different modes and receiver schemes considered. We perform the throughput analysis via some numerical examples.

\section{A. Numerical results}

In figure (4), we plot the total achievable throughput in $C_{0}$ as a function of the cell size $L$ which is obtained using equation (7), (10). We consider two cases, the first case when all the cells deployed use the same frequency and the second case where we use a reuse factor of 3. i.e, every third cell uses the same frequency. We compute the throughput for the matched filter as well as the multi-user detection scheme 
in both single frequency and frequency reuse. We plot the throughput as a function of $L$ in figure (4) for $\alpha=2$. As expected, the throughput increases with $L$ and multi-user detection performs better than the matched filter.

Next, we look at the total throughput density achievable in $C_{0}$ as a function of $L$. For the single frequency case, when the BS receiver uses a matched filter $\left(S_{5} \_M F\right)$, we note that the numerator in the throughput density (equation (8)) depends on $L$; it represents the useful power density received. By Lemma 1, we know that the optimal cell size that maximizes the throughput density, $L^{*} \rightarrow 0$. Figure (3) plots the power density as a function of $L$. We observe that the power density decreases with $L$ and is maximum when $L^{*} \rightarrow 0$. Thus we can conclude that the throughput density is maximized by taking base stations as dense as possible for a BS receiver which uses a matched filter. With frequency reuse, the throughput density also depends upon $L$ via the total received power (see equations (4), (8)). Hence, we directly compute the throughput density in this case.

As before, we consider the two cases described previously. We plot the throughput density as a function of $L$ in figure (5), for $\alpha=2$. We also plot the throughput density for a single frequency with a matched filter (SF_MF), frequency reuse with a matched filter (FR_MF), single frequency with multi-user detection (SF_MD) and frequency reuse with multiuser detection (FR_MD) in the same figure.

By Lemma 2, we would expect that the cell size which maximizes the throughput density, $L^{*}>0$, for the BS receiver which employs multi-user detection. From the numerical example we conducted (figure (5) ), we indeed see that $L^{*}>0$ and in fact, there is a unique optimal $L$ for a certain frequency reuse which maximizes the throughput density. The effect is more pronounced in the frequency reuse case. Also, we achieve a higher throughput density for smaller cell sizes for the more practical case of $\alpha=4$ in contrast to $\alpha=2$.

\section{B. Optimizing the cell size}

Matched filter: We see from figure (5) that in the matched filter case, the throughput density is maximized for smaller cell sizes. However there is a cost for deploying base stations. So it is more natural to pose the problem of maximizing

$$
J(L)=\frac{1}{L}\left(c_{1} \Theta(L)-c_{2}\right)
$$

where $c_{1} / L$ is the revenue per throughput density and $c_{2}$ is the cost of each BS.

We note from figure (10) that the throughput density is not sensitive at all to the reuse parameter $m$ in the matched filter case. (the cell size, $L^{*}$, which maximizes the throughput density is always equal to the smallest $L$ considered in the numerical analysis). Also, note that the optimization is over the integers and $L$ is typically not larger than a few hundreds. This allows one to solve the optimization problem in a very short time even if exhaustive search is used.

Multi-user detection: For a BS which employs multi-user detection, we observe from numerical analysis (see figure (10)) that $L^{*}$ decreases as the reuse factor $m$ increases. So, as the reuse factor increases, one would prefer to reduce the cell size in order to maximize throughput density (densify base stations). However, this increases deployment costs. Hence, our problem is to find an optimal cell size which balances the throughput and deployment costs over the reuse factors of interest. Assuming that the deployment cost is proportional to the frequency reuse factor $m$, we can formulate this problem as:

$$
J(L, m)=\frac{1}{L}\left(c_{1} \Theta(L)-c_{2}-m c_{3}\right)
$$

as before, $c_{1} / L$ is the revenue per throughput density and $c_{2}$ is the cost of each BS. The additional cost $m c_{3}$ is incurred due to reuse co-ordination where $m$ is the reuse factor and $c_{3}$ is the cost per reuse.

\section{INDOOR ANALYSIS}

\section{A. BS located inside the building}

Next, we consider the cell of interest to be enclosed within a wall. All the interferers are located outside of the wall. We use our earlier received power and throughput computation to analyze the performance of the desired cell for both the matched filter as well as the multi-user detection schemes. Here again, we consider single frequency as well as frequency reuse, first in the $1 \mathrm{D}$ case. We add to the gain, a constant multiplication term to take into account the attenuation due to penetration through walls (refer [7], [8], [9] for some example values).

Thus the throughput density equations (8) and (11) change as $\Psi_{M F}(L)=\frac{P_{b s}^{C_{0}}(L) / L}{\sigma^{2}+\eta P_{b s}^{t o t}+(1-\eta) P_{b s}^{C_{0}}(L)}$. and $\Psi_{M D}(L)=$ $\frac{1}{L} \log \left(1+\frac{P_{b s}^{C_{0}}(L)}{\sigma^{2}+\eta\left(P_{b s}^{t o t}-P_{b s}^{C_{0}}(L)\right)}\right)$, where $\eta$ is the wall attenuation factor.

We plot the numerical results in figure (7) for attenuation $\eta=12 d B$

\section{Observations:}

- When the interference is attenuated, there does not exist an optimal cell size which maximizes the throughput density for both the matched filter as well as multiuser detection schemes in single frequency and frequency reuse respectively.

- Throughput density increases with path-loss $\alpha$.

- Throughput density increases with attenuation $\eta$.

\section{B. BS located outside the building}

Another interesting case is the one where we assume that the serving $\mathrm{BS}$ is located outside of the wall, but geometrically within the cell (example, BS mounted on top of the roof, served mobiles inside the building (cell)). The throughput density equations (8) and (11) change as $\Psi_{M F}(L)=\frac{\eta P_{b s}^{C_{0}}(L) / L}{\sigma^{2}+P_{b s}^{t o t}-(1-\eta) P_{b s}^{C_{0}}(L)}$. and $\Psi_{M D}(L)=$ $\frac{1}{L} \log \left(1+\frac{\eta P_{b s}^{C_{0}}(L)}{\sigma^{2}+P_{b s}^{t o t}-P_{b s}^{C_{0}}(L)}\right)$. 
We plot the throughput density as a function of the cell size in figure (8) for $12 \mathrm{~dB}$ attenuation.

Observations:

- For both the matched filter and multi-user detection, the maximum throughput is achieved for a certain optimal cell size.

- As expected, the achievable throughput density increases with path-loss $\alpha$.

- The optimal cell size decreases as path-loss $\alpha$ increases.

- The advantage of multi-user detection over the matched filter diminishes as the attenuation $\eta$ increases.

- The optimal cell size increases as the attenuation $\eta$ increases.

Remarks: From the above two cases, it is interesting to note that the placement of the BS matters, when the cell of interest is located indoors.

\section{DIMENSION 2}

In this section, we want to compute the optimal cell sizes in two dimension (2D). We begin our study with the power and throughput computation similar to the 1D case.

- The total power received for $\alpha>2, P_{b s}^{t o t}=\int_{0}^{\infty} 2 \pi x(1+$ $\left.x^{2}\right)^{-\alpha / 2} \mathrm{~d} x=\frac{2 \pi}{\alpha-2}$. Whereas, for $\alpha \leq 2$ the total received power is infinite.

- Consider a cell $C_{0}$ centered at the origin with radius $L$. The power received at the origin from mobiles within the cell is $P_{b s}^{C 0}=\int_{0}^{L} 2 \pi x\left(1+x^{2}\right)^{-\alpha / 2} \mathrm{~d} x=\frac{2 \pi}{\alpha-2}(1-(1+$ $\left.\left.L^{2}\right)^{1-\alpha / 2}\right)$

- The total throughput achievable at a cell when decoding each mobile using the matched filter (considering all the rest as noise) is $T h p_{C}^{a d a p t-f i l t e r}=\frac{1-\left(1+L^{2}\right)^{1-\alpha / 2}}{\sigma^{2} \frac{2-\alpha}{2 \pi}+1}$

- The total throughput achievable at a cell when multi-user detection is used (considering all the mobiles out of the cell as noise) is $T h p_{C}^{\text {Mult-Acc }}=$ $\log \left(1+\frac{\left.1-\left(1+L^{2}\right)^{1-\alpha / 2}\right)}{\sigma^{2} \frac{2-\alpha}{2 \pi}+\left(1+L^{2}\right)^{1-\alpha / 2}}\right)$.

For the two dimensional case, we consider frequency reuse only.

\section{A. A simple approximation to the hexagonal grid}

In this case, we approximate the hexagon cells with virtual circles (similar to the examples used in [2], [4] or [5]). Given that we consider a circle with radius $L$, we can construct a hexagon with side $L^{\prime}$, such that the area of the hexagon and the circle are the same [14]. One can easily see that $L^{\prime}=$ $\left(\sqrt{\frac{2 \pi}{3 \sqrt{3}}}\right) L$. For example, if $L=1, L^{\prime}=1.0996 L$. Also, the number of hexagons grow by 6 for each tier.

The infinite hexagonal grid representation is shown in figure (11). The inner most hexagon is the cell of interest, where we desire to compute the throughput density. We consider a reuse 4 scenario. The subsequent hexagonal cells surrounding the cell of interest has the frequency allocation as shown in figure

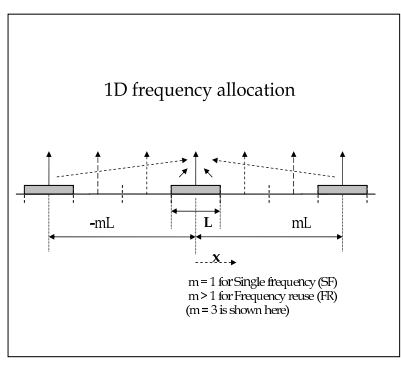

Fig. 1. Frequency allocation in 1D.

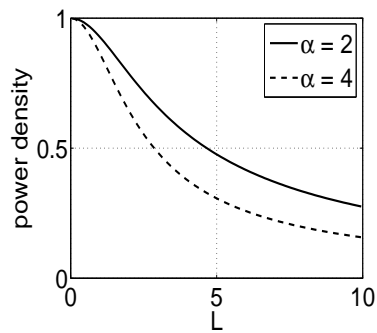

Fig. 3. Total power density from $C_{0}$ vs $L(\alpha=2,4$; single frequency, matched filter).

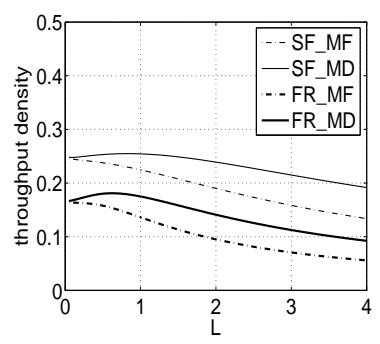

Fig. 5. Throughput density vs $L$ $(\alpha=2)$.

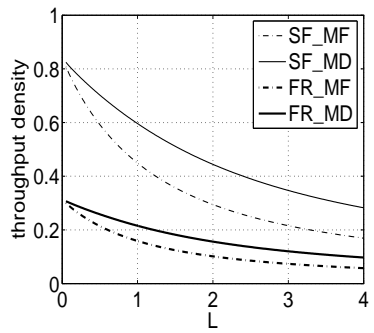

Fig. 7. Throughput density $v s L$ $(\alpha=2$, wall attenuation $12 d B$, BS indoors).

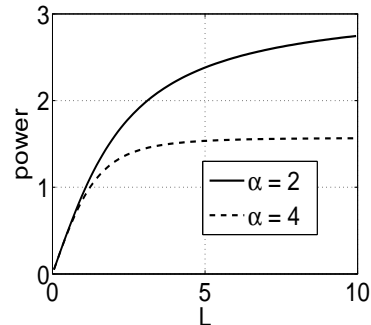

Fig. 2. Total power received at the $\mathrm{BS}$ in $C_{0}$ vs $L$ ( $\alpha=2,4$; single frequency).

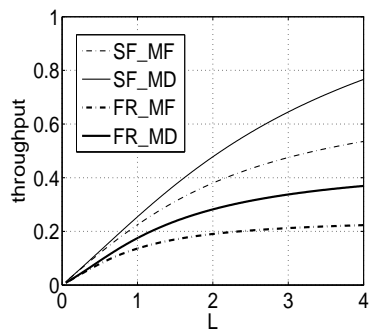

Fig. 4. Total throughput of cell $C_{0}$ vs $L(\alpha=2)$.

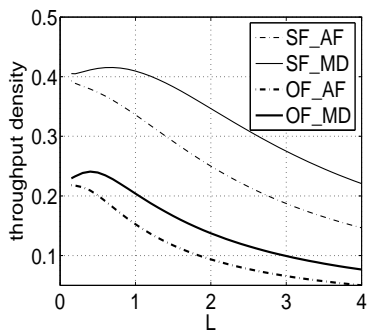

Fig. 6. Throughput density vs $L$ $(\alpha=4)$.

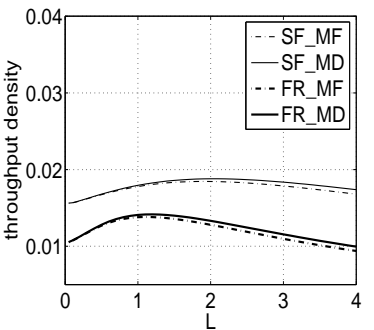

Fig. 8. Throughput density vs $L$ $(\alpha=2$, wall attenuation $12 d B$, BS outdoors). 


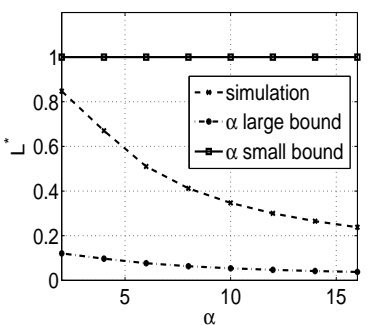

Fig. 9. Optimal cell size $L^{*}$ vs path-loss factor $\alpha$ (reuse factor $m=1$ )

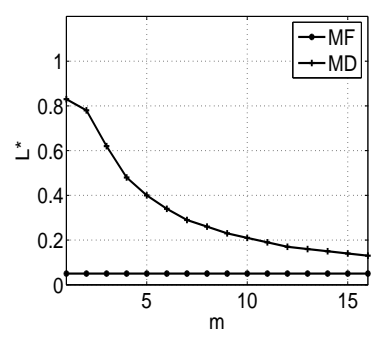

Fig. 10. Optimal cell size $L^{*}$ $v s$ reuse factor $m$ (path-loss factor $\alpha=2)$.
(11). As we see, every alternate tier repeats the frequency of the inner-most cell, thus contributing to interference to the cell of interest. We can also observe that the amount of interference is $1 / 2$ of the total contribution from this tier of hexagons.

The interference power from this annular ring is given by

$$
\begin{array}{r}
P_{b s}^{C(j)}=\frac{1}{m} \frac{1}{2} \frac{2 \pi}{\alpha-2}\left\{\left(\left(1+\left(a_{j} L\right)^{2}\right)^{1-\alpha / 2}\right)\right. \\
\left.-\left(\left(1+\left(b_{j} L\right)^{2}\right)^{1-\alpha / 2}\right)\right\}
\end{array}
$$

where $a_{j}=\sqrt{(2 j) 6+1}, b_{j}=\sqrt{(2 j-1) 6+1}$ and $j$ takes the value $1,2,3 \ldots$ representing the power from the $j^{\text {th }}$ odd hexagonal tier. Note that $m$ is the reuse factor.

\section{B. A more precise approximation for the 2-D hexagonal grid}

In this section, we propose an alternate approach to derive a more precise approximation for the 2-D hexagonal grid and compare it with the previously used approximation. In figure (11), every tier of hexagons around the cell of interest is enclosed by an hexagon (shown dotted), whose side $L^{\prime \prime}=\left(\frac{2}{\sqrt{3}}+N \sqrt{3}\right) L^{\prime}$. Where, $\mathrm{N}$ is the index of the hexagonal tier. As before, we replace these hexagons with annular rings, where, the radius of the annular rings is related to the enclosed hexagons by $L^{\prime \prime}=\left(\sqrt{\frac{2 \pi}{3 \sqrt{3}}}\right) L$. Using these relationships, we can see that the radii of the equivalent annular ring's for this model grows as $\frac{1}{\sqrt{3}}(2 r, 5 r, 8 r, \ldots($ Method $B)$ as compared to $r, \sqrt{7} r, \sqrt{13} r, \sqrt{19} r \ldots$ (Method $A)$ in the previous case.

The numerical results are shown in figure (12). We conclude from this new approximation for the choice of radii in the earlier case Method $A$ was conservative. Thus using Method $B$ would result in a lower throughput density.

\section{Throughput density with reuse}

In this section, we would like to compare how the throughput density changes as as function of the reuse factor. We use the 2-D hexagonal model proposed (Method $B$ ) in our numerical simulations. For this study, we construct hexagonal grid with 10-tiers around the cell of interest. This would encompass 331 cells in total. So we would be considering interference from a possible 330 cells surrounding our cell of interest. For the numerical analysis, we use reuse 1, 2, 3, 4 and 7.

The amount of interference contributed from each tier for different reuse factors are listed in the table II.

Now equation (19) is modified to accommodate the reuse factor, $m$ and re-written as shown

$$
\begin{array}{r}
P_{b s}^{C(j)}=\frac{1}{m} c_{j} \frac{2 \pi}{\alpha-2}\left\{\left(\left(1+\left(a_{j} L\right)^{2}\right)^{1-\alpha / 2}\right)\right. \\
\left.-\left(\left(1+\left(b_{j} L\right)^{2}\right)^{1-\alpha / 2}\right)\right\}
\end{array}
$$

now, $a_{j}=\sqrt{(3 j)+2}, b_{j}=\sqrt{(3(j-1)+2}$, to make it generic to accommodate all possible reuse factors and $j$, the tier number goes from $1,2, . ., 10 . c_{j}$ is the interference contribution from the $j^{\text {th }}$ tier (ex. from table II, for reuse 4, the interference contribution is $1 / 2$ for every odd tier). We use equation (20), to compute the throughput density.

We see from figure (13) that the throughput density increases with the reuse factor. The other interesting point to note is that the throughput density for higher reuse does not fall rapidly as compared to the lower reuse factors.

\section{Indoor analysis}

For our next analysis, we assume that the cell of interest is located indoors and that the walls offer an attenuation of $\eta=12 d B$ to the interferer's. figure (14) captures this case for all reuse factors. We would also like to know what is the benefit or gain in throughput density when the interferer's are attenuated. This can be captured by including the case of reuse factor 4 from the previous numerical analysis. We see that for smaller cell sizes, this is almost a factor of 2 (see the region ellipse in the figure). But, one loses this advantage as the cell sizes tend to increase. Finally, we look at the case of the cell of interest located indoors, but the BS located outside, say, mounted on top of the roof. Now the signals from mobiles inside the building are attenuated by $\eta=12 d B$. figure (15) captures this analysis. The reduction in throughput density in such a case is very drastic as one can observe for the case of reuse factor 4 . The benefit of reuse hardly seems to help in such situations (see the ellipse in the figure). In conclusion, we see that the gain in throughput density when the BS is located indoors is more than compensated when one moves it outside. This would mean that one would need to plan appropriate BS placement, depending on the nature of user's demand.

\section{COVERAGE AND CAPACITY}

Next, we want to study the trade-off between capacity and coverage. We assume that some portion towards the periphery of the cell is not covered by the BS and hence these mobiles are switched off (in power saving mode). We want to look at the throughput per mobile as a function of coverage and the total capacity achievable at the cell, again as a function of coverage. 
TABLE II

INTERFERENCE CONTRIBUTION FOR DIFFERENT REUSE FACTORS.

\begin{tabular}{|c|cccccccccc|}
\hline tier no. & 1 & 2 & 3 & 4 & 5 & 6 & 7 & 8 & 9 & 10 \\
\hline reuse 1 & 1 & 1 & 1 & 1 & 1 & 1 & 1 & 1 & 1 & 1 \\
reuse 2 & $1 / 3$ & $2 / 3$ & $1 / 3$ & $2 / 3$ & $1 / 3$ & $2 / 3$ & $1 / 3$ & $2 / 3$ & $1 / 3$ & $2 / 3$ \\
reuse 3 & 0 & $1 / 2$ & $1 / 3$ & $1 / 4$ & $2 / 5$ & $2 / 6$ & $2 / 7$ & $3 / 8$ & $3 / 9$ & $3 / 10$ \\
reuse 4 & 0 & $1 / 2$ & 0 & $1 / 2$ & 0 & $1 / 2$ & 0 & $1 / 2$ & 0 & $1 / 2$ \\
reuse 7 & 0 & 0 & $1 / 3$ & 0 & $1 / 5$ & $1 / 6$ & $1 / 7$ & $1 / 8$ & $1 / 9$ & $2 / 10$ \\
\hline
\end{tabular}

\section{A. Coverage and capacity in a single cell}

We assume power control. i.e, mobiles at the boundary of the coverage area (distance $x$ from BS) transmit with power $P$ and the received power at the BS is

$$
p=P\left(1+x^{2}\right)^{-\alpha / 2} .
$$

Since we assume power control, any mobile within this coverage area will transmit with a power $P^{\prime}$ lesser than $P$, such that the received power at the BS will always satisfy equation (21). The throughput per mobile can be computed as

$$
d \theta(x)=\frac{p}{1+\int_{0}^{x} p d x}=\frac{p}{1+p x}
$$

while, the capacity of the cell as a function of coverage $x$ can be computed as

$$
C(x)=x d \theta(x)=\frac{p x}{1+p x}
$$

We observe that (figure (16)) the capacity of the cell increases with increase in coverage.

\section{B. Coverage and capacity on a line segment (1D)}

Next, we want to extend the argument for the entire line segment. For the ease of analysis, we assume no power control and as before, the mobiles, which are not in the coverage area are in a power-down state. In this context, we want to analyze how the throughput density changes as a function of coverage for both multi-user detection (MD) and the matched filter (MF) case. We consider frequency reuse of 3 .

We use equations (2), (3), (4) and (5) and account for the coverage by replacing $L$ with $L+\delta L$ or $L-\delta L$ appropriately. In our numerical examples, we compare full coverage with 75 $\%$ coverage.

Figure (17) shows the throughput density. We observe that for the multi-user detection scheme, the full as well as the 75 $\%$ coverage exhibits certain cell size for maximum throughput density, while in the matched filter case, the throughput density is maximized by densifying the cells. Hence, we conclude that coverage does not alter the behavior of achievable maximum throughput, albeit at a different cell size for the multi-user detection case.

We observe that for small cell sizes, the throughput density (refer to figure (17)) is proportional to the coverage. But, as cell size increases, the throughput density for different coverage tends to converge.

\section{Coverage and capacity in two dimension}

Next, we look at coverage and capacity in 2-dimension. As before, we have an infinite hexagonal grid and our analysis assumes interference coming from hexagonal rings surrounding the cell of interest. Here again, we consider full and 75 $\%$ cell coverage and compute throughput density for different values of $\alpha$

The following are note-worthy observations (see figure (18)).

1. The achievable throughput density falls very sharply in the vicinity of a small cell radii, irrespective of the coverage.

2 . We achieve a maximum throughput density proportional to the coverage. i.e for example, the MD scheme with $75 \%$ coverage achieves about $75 \%$ of maximum throughput density of the full coverage case at small cell radii. But, as the cell size increases, the achieve throughput density with $75 \%$ coverage starts moving closer to the full coverage case.

\section{CONCLUSIONS}

In our paper, we characterize the throughput achievable at the BS as a function of the cell size. This study helps us to find an optimal cell size which maximizes the achievable throughput. We derived explicit expressions for power and throughput for both single frequency and frequency reuse with different receiver configurations and numerically analyze the impact of the cell size on throughput for various 1D, 2D models in both indoor and outdoor scenarios. Our first cut analysis used free space path loss and we did not consider the impact of shadowing, fading, etc. Our analysis shows that the throughput achievable is not always maximized by densifying the base-stations (BS), but rather depends on a case to case basis on factors like deployment (1D, 2D, indoor, outdoor), frequency reuse, etc. As an application of our model, one can also extend it to self organize networks when the base stations are also mobile. Our future work would focus on incorporating the effects of shadowing, fading and mobility into the model and will search for efficient ways to evaluate their performance, moving a step closer to realize optimal pico-cell networks.

\section{ACKNOWLEDGMENT}

This work was done in the framework of the INRIA and Alcatel-Lucent Bell Labs Joint Research Lab on Self Organized Networks. The work of Mrouane Debbah is done within the Alcatel-lucent chair on Flexible Radio. 


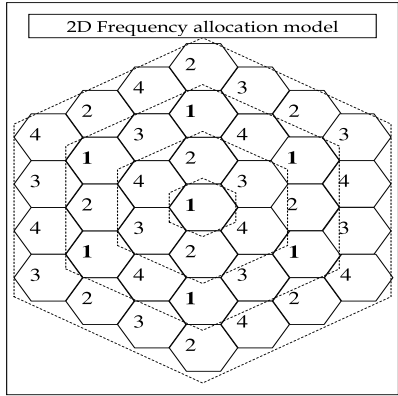

Fig. 11. Frequency allocation in 2D.

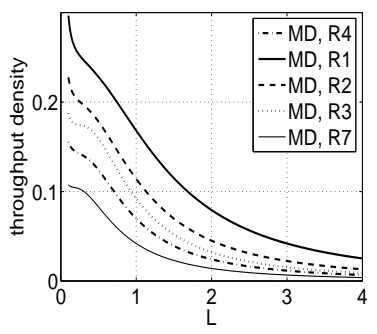

Fig. 13. Throughput density vs $L$ for $\alpha=4.1$ and different reuse factors.

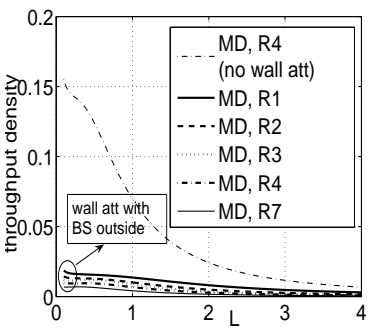

Fig. 15. Throughput density $v s$ $L$ for $\alpha=4.1$ and different reuse factors (indoor cell, BS outdoors).

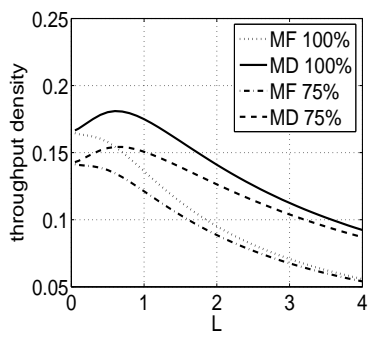

Fig. 17. Throughput density vs $L$ for $100 \%$ and $75 \%$ cell coverage ( $\alpha=2,1 \mathrm{D})$.

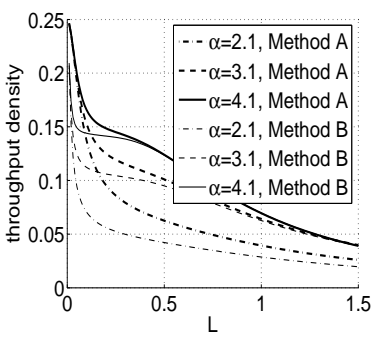

Fig. 12. Throughput density vs $L$ for different $\alpha$ and decoding schemes comparing MethodA with MethodB.

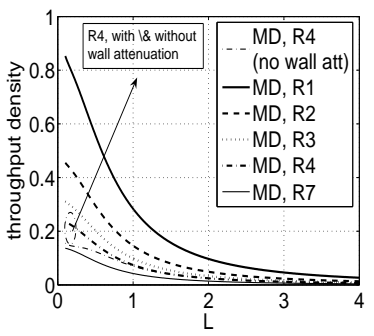

Fig. 14. Throughput density vs $L$ for $\alpha=4.1$ and different reuse factors (BS indoors).

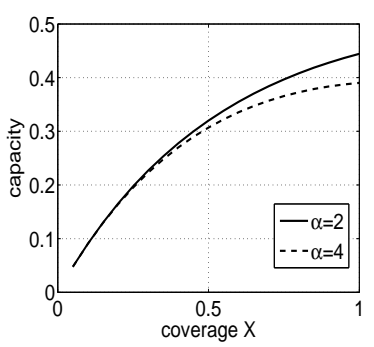

Fig. 16. Capacity vs cell coverage $x$ for $\alpha=2,4$.

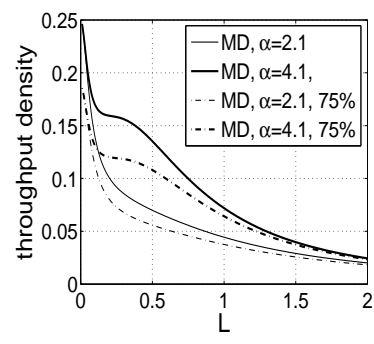

Fig. 18. Throughput density vs $L$ for $100 \%$ and $75 \%$ cell coverage ( $\alpha=4.1$, multi-user detection, 2D).

\section{REFERENCES}

[1] J. M. Kelif and E. Altman, Downlink Fluid Model of CDMA Networks, In Proc. of IEEE VTC Spring, May 2005

[2] N. Bonneau, M. Debbah, E. Altman and G. Caire, Spectral Efficiency of CDMA Uplink Cellular Networks, Proceedings of IEEE International Conference on Acoustics, Speech and Signal Processing, Philadelphia, USA, March 2005.

[3] N. Bonneau, M. Debbah, and E. Altman, Spectral Efficiency of CDMA

Downlink Cellular Networks with Matched Filter, EURASIP Journal on Wireless Communications and Networking, 2005.

[4] Zorzi, M. On the analytical computation of the interference statistics with applications to the performance evaluation of mobile radio systems, Communications, IEEE Transactions on, Volume 45, Issue 1, Jan 1997, Page(s): 103-109

[5] Philippe Godlewski, Masood Maqbool, Marceau Coupechoux, and Jean-Marc Kelif, Analytical Evaluation of Various Frequency Reuse Schemes in Cellular OFDMA Networks ValueTools 2008, Performance Evaluation.

[6] D. Stamatelos and A. Ephremides, Spectral Efficiency and Optimal Base Placement for Indoor Wireless Networks IEEE JSAC, Volume 14, Issue 4, May 1996, Page(s): 651-661

[7] Siqueira, G.L, Vasquez, E.V, Gomes, R.A, Sampaio, C.B, Costa, V.C.F and Socorro, M.A, Propagation measurements for indoor mobile picocell coverage, Microwave and Optoelectronics Conference, Aug 1997

[8] Robert. A, Dinesh. T, Xinrong. Li, Indoor propogation modeling at 2.4 GHz for 1EEE 802.11 networks International conference on wireless and optical communication, Jul 2006

[9] Ding Xu, Jianhua Zhang, Xinying Gao, Ping Zhang and Yufei Wu, Indoor Office Propagation Measurements and Path Loss Models at $5.25 \mathrm{GHz}$, VTC, Sept 2007, Page(s): 844-848

[10] Theodore Rappaport, Wireless Communications: Principles and Practice (2nd Edition) Prentice Hall 2001

[11] Sergio Verdu, Multiuser Detection Cambridge press 2003

[12] Gregory Davi, Using picocells to build high-throughput 802.11 networks RF Design, Jul 2004

[13] Picocell Mesh: Bringing Low-Cost Coverage, Capacity and Symmetry to Mobile WiMAX A Tropos Networks white paper

[14] Mohamed Kadhem KARRAY, Analytic evaluation of wireless cellular networks performance by a spatial Markov process accounting for their geometry, dynamics and control schemes, Doctoral thesis, Sep 2007, ENST 